\title{
Sur la non-linéarité des fonctions booléennes
}

\author{
par \\ FranÇOIS RODIER (Marseille)
}

\section{INTRODUCTION}

Les fonctions booléennes sur l'espace $\mathbb{F}_{2}^{m}$ interviennent aussi bien dans la théorie des codes correcteurs d'erreurs (par exemple dans les codes de ReedMuller), qu'en cryptographie pour réaliser des systèmes de chiffrement à clef secrète.

Dans ces deux cas, les propriétés des systèmes ainsi construits dépendent en particulier de la non-linéarité d'une fonction booléenne, concept que je définirai précisément plus loin (\$2.3). La non-linéarité est liée au rayon de recouvrement des codes de Reed-Muller. C'est aussi un paramètre cryptographique important : dans leur article [11], F. Chabaud et S. Vaudenay montrent que la non-linéarité est un critère important de résistance aux attaques différentielles et linéaires; dans sa thèse, C. Fontaine [16] met en valeur l'importance de la non-linéarité en cryptographie pour plusieurs systèmes de chiffrement.

Il est utile de pouvoir disposer de fonctions booléennes ayant la plus grande non-linéarité possible, comme l'ont montré W. Meier et O. Staffelbach dans [22], ainsi que K. Nyberg dans [24]. Ces fonctions ont été étudiées dans le cas où $m$ est pair, sous le nom de fonctions "courbes" (cf. J. Dillon [13]). Leur degré de non-linéarité est alors bien connu, on sait construire plusieurs séries de fonctions courbes, mais on ne connaît encore ni leur nombre, ni leur classification (cf. les travaux de C. Carlet, en particulier l'article de C. Carlet et P. Guillot [9], ou de C. Carlet et A. Klapper [10]). Dans le cas où $m$ est impair, la situation est bien différente : on ne connaît alors la valeur de la non-linéarité maximale que pour quelques valeurs de $m$, et on n'a qu'une conjecture pour les autres valeurs (voir le mémoire d'habilitation de P. Langevin [19]).

2000 Mathematics Subject Classification: Primary 11T71; Secondary 06E30, 42A05, 94B75.

Key words and phrases: covering radius, error-correcting code, cryptography, nonlinearity, boolean function, random polynomial, Fourier transform. 
Dans cet article, je veux montrer que pour traiter les fonctions booléennes, on peut s'inspirer de la théorie des polynômes aléatoires qui a été un sujet d'étude depuis les travaux de Paley et Zygmund. En effet, le problème de la recherche du maximum du degré de non-linéarité revient à minimiser la transformée de Fourier de fonctions booléennes. C'est un problème analogue aux séries de Fourier sur un tore, où l'on cherche à minimiser la transformée de Fourier des fonctions sur $\mathbb{Z}$ prenant les valeurs \pm 1 pour un ensemble fini (et 0 ailleurs), ce qui revient à chercher à minimiser les valeurs des polynômes à coefficient \pm 1 (polynômes aléatoires) sur l'ensemble des nombres complexes de module 1 .

Dans cet article, on s'inspire des travaux de R. Salem et A. Zygmund [27] et de J.-P. Kahane [17] sur les polynômes aléatoires, en les transposant sur les fonctions booléennes. On trouve ainsi une évaluation de la moyenne des normes dans $L_{\infty}$ des transformées de Fourier des fonctions booléennes, qui n'est pas trop éloignée de sa valeur minimale théorique, $2^{m / 2}$. Cela donne une évaluation de la moyenne des degrés de non-linéarité de ces fonctions. On retrouve en particulier le fait que la plupart des fonctions booléennes ont une grande non-linéarité, un résultat mis en évidence récemment par D. Olejár et M. Stanek [25] et C. Carlet [7, 8] (cf. théorème 4.1). Le résultat que j'ai démontré implique en outre que presque toutes les fonctions booléennes ont un non-linéarité voisine d'une même valeur. Cette propriété est illustrée par exemple par les diagrammes de [1], qui exhibent la non-linéarité de fonctions booléennes en vue de la construction de boîtes de substitutions (s-boxes) utilisées dans les chiffrements par blocs, ou de l'étude statistique de [16, chapitre 6].

De plus, en transposant une étude de D. Newman et J. Byrnes [23] sur les normes dans $L_{4}$ des polynômes, nous avons été amenés à étudier une conjecture sur la norme dans $L_{4}$ des transformées de Fourier de fonctions booléennes. On retrouve ainsi le critère de la "somme des carrés", relié au critère de propagation, pour les fonctions booléennes. Ce critère a été étudié par X. M. Zhang et Y. L. Zheng [29], ou par P. Stănică [28]. Son rapport avec la non-linéarité a été étudié par A. Canteaut et al. [6].

\section{PRÉLIMINAIRES}

2.1. Fonctions booléennes. Soit $m$ un entier positif et $q=2^{m}$.

DÉFINITION 2.1. Une fonction booléenne à $m$ variables est une application de l'espace $V_{m}=\mathbb{F}_{2}^{m}$ dans $\mathbb{F}_{2}$.

Une fonction booléenne est linéaire si c'est une forme linéaire sur l'espace vectoriel $\mathbb{F}_{2}^{m}$. Elle est dite affine si elle est égale à une fonction linéaire à une constante près. 


\subsection{Rayon de recouvrement du code du Reed-Muller du pre- mier ordre et amplitude spectrale}

DÉFInItion 2.2. L'amplitude spectrale de la fonction booléenne $g$ est égale à

$$
S(g)=\sup _{v \in V_{m}}\left|\sum_{x \in V_{m}}(-1)^{g(x)+v \cdot x}\right|
$$

où $v \cdot x$ note le produit scalaire usuel dans $V_{m}$. C'est le maximum de la transformée de Fourier de $(-1)^{g}$.

Cette amplitude spectrale est reliée au rayon de recouvrement du code du Reed-Muller. En effet, le code de Reed et Muller $\mathcal{R}_{m}$ d'ordre 1 sur $V_{m}$ est l'espace vectoriel des fonctions booléennes affines sur $V_{m}$. Le rayon de recouvrement $r_{m}$ du code est le plus petit entier tel que chaque vecteur de longueur $2^{m}$ (c'est-à-dire chaque fonction $V_{m} \rightarrow \mathbb{F}_{2}$ ) est à une distance (de Hamming) d'un mot de code de $\mathcal{R}_{m}$ au plus égale à $r_{m}$. On vérifie que

$$
r_{m}=2^{m-1}-\frac{1}{2} \mu_{m} \quad \text { où } \mu_{m}=\inf _{g} S(g)
$$

où $g$ est une fonction $V_{m} \rightarrow \mathbb{F}_{2}$ et où $S(g)$ est l'amplitude spectrale de $g$.

\subsection{Non-linéarité}

DÉfinition 2.3. On appelle degré de non-linéarité d'une fonction booléenne $g$ à $m$ variables, et on le note $\mathrm{nl}(g)$, la distance qui la sépare de l'ensemble des fonctions affines à $m$ variables :

$$
\operatorname{nl}(g)=\min _{h \text { affine }} d(g, h)
$$

où $d$ est la distance de Hamming.

Proposition 2.1. Soit $g$ une fonction booléenne à $m$ variables. Son degré de non-linéarité est égal à

$$
\operatorname{nl}(g)=2^{m-1}-\frac{1}{2} S(g) .
$$

C'est la même démonstration que pour le rayon de recouvrement d'un code de Reed et Muller.

2.4. Résultats connus, conjecture. Le rayon de recouvrement du code du Reed-Muller du premier ordre est bien connu pour une dimension $m$ paire : $\mu_{m}$ vaut $2^{m / 2}$. Pour $m$ impair, on n'a connu longtemps qu'un encadrement de $\mu_{m}: 2^{m / 2} \leq \mu_{m} \leq 2^{(m+1) / 2}$. En 1983, N. Patterson et D. Wiedemann [26] ont montré que l'on peut faire mieux pour $\mathcal{R}_{15}$ en exhibant une fonction booléenne telle que

$$
\mu_{15} \leq \frac{27}{32} \sqrt{2} 2^{15 / 2}
$$


Ils en ont déduit que si $m$ est impair et plus grand que 15 , on a

$$
\mu_{m} \leq \frac{27}{32} \sqrt{2} 2^{m / 2}
$$

Ils ont conjecturé que $\mu_{m} \sim 2^{m / 2}$.

Remplaçons la fonction booléenne $g$ par son exponentielle

$$
f(x)= \begin{cases}1 & \text { si } g(x)=0, \\ -1 & \text { si } g(x)=1 .\end{cases}
$$

On définit la transformée de Fourier de $f$ par $\widehat{f}(\chi)=\sum_{V_{m}} f(x) \chi(x)$ où $\chi$ est un caractère de $V_{m}$, c'est-à-dire ici un homomorphisme de $V_{m}$ dans $\{ \pm 1\}$, de telle sorte que $S(g)=\|\widehat{f}\|_{\infty}$.

La conjecture de Patterson et Wiedemann se réécrit alors comme suit :

Conjecture 2.1. Si $f$ décrit l'espace des fonctions de $V_{m}$ dans $\{ \pm 1\}$, on $a$

$$
\liminf _{m} \frac{\|\widehat{f}\|_{\infty}}{2^{m / 2}}=1
$$

2.4.1. Cas du groupe des nombres complexes de module égal à 1. Ce problème a un analogue avec les séries de Fourier sur le tore (c'est-à-dire sur le groupe $\mathbb{U}$ des nombres complexes de module égal à 1$)$.

En effet la définition de l'amplitude spectrale peut s'écrire

$$
S(g)=\sup _{v \in V_{m}}\left|\sum_{x \in V_{m}} f(x)(-1)^{v \cdot x}\right| .
$$

Remplaçons dans cette relation les fonctions

$$
V_{m} \rightarrow\{ \pm 1\} \subset \mathbb{C}^{*}, \quad x \mapsto(-1)^{v \cdot x}
$$

pour $v \in V_{m}$, qui sont des caractères de $V_{m}$, par des caractères du tore $\mathbb{U}$ de la forme

$$
\mathbb{U} \rightarrow \mathbb{C}^{*}, \quad z \mapsto z^{s}
$$

pour $s \in \mathbb{Z}$, et les fonctions booléennes

$$
V_{m} \rightarrow\{ \pm 1\}, \quad x \mapsto f(x)
$$

par les suites

$$
\mathbb{Z} \rightarrow\{ \pm 1\}, \quad s \mapsto a_{s} .
$$

La conjecture 2.1 peut se réécrire

$$
\liminf _{n} \frac{\left\|\sum_{s=0}^{n} a_{s} z^{s}\right\|_{\infty}}{\sqrt{n}}=1
$$

où l'infimum porte sur l'ensemble des suite $a_{s}= \pm 1$. Autrement dit, il existerait une suite de polynômes $P_{n}(z)$ et une suite de nombres positifs $\varepsilon_{n}$ 
tendant vers zéro telles que pour tout $|z|=1,\left|P_{n}(z)\right| \leq\left(1+\varepsilon_{n}\right) \sqrt{n}$, où $P_{n}(z)$ est un polynôme aléatoire de la forme

$$
P_{n}(z)=\sum_{s=0}^{n} a_{s} z^{s} \quad \text { avec } a_{s}= \pm 1 .
$$

Ce problème a été posé par divers auteurs comme J. E. Littlewood [21], et P. Erdős [15] qui a conjecturé qu'au contraire il existe $\delta>1$ tel que quel que soient l'entier $n$ et le nombre complexe $z$ de module 1 , on ait $\left|P_{n}(z)\right| \geq \delta \sqrt{n}$. Kahane $([17])$ a résolu un problème analogue dans le cas où les coefficients complexes $a_{s}$ peuvent prendre toutes les valeurs de module 1, mais rien n'a été fait pour le problème initial, où les coefficients $a_{s}$ sont égaux à \pm 1 . Dans ce livre, Kahane utilise pour résoudre ce problème des exponentielles de la forme $e^{\pi i n^{2} / a}$, donc des exponentielles de formes quadratiques en $n$. Il fabrique avec cela un polynôme $P_{n}(z)$ qui résout presque le problème. Il ajuste ensuite ce polynôme en utilisant un argument de probabilité. Transposé dans notre cas, ces exponentielles de formes quadratiques en $n$ correspondent à des fonctions booléennes de la forme $(-1)^{Q(x)}$ où $Q(x)$ est une forme quadratique en $x \in V_{m}$. Mais ces fonctions booléennes ne donnent pas de résultat complet pour les dimensions $m$ impaires. Elles ont une amplitude spectrale au mieux de la forme $2^{m / 2} \sqrt{2}$.

\section{L'ESPACE DES FONCTIONS BOOLÉENNES À UNE INFINITÉ DE VARIABLES}

Pour étudier asymptotiquement les fonctions booléennes, en particulier pour définir la notion de propriété presque sûre, on aura besoin de la notion de fonction booléenne à une infinité de variables.

On rappelle que $V_{m}=\mathbb{F}_{2}^{m}$. On définit une application de transition entre $V_{m}$ et $V_{m+1}$ par

$$
\phi_{m}: V_{m} \rightarrow V_{m+1}, \quad\left(x_{1}, \ldots, x_{m}\right) \mapsto\left(x_{1}, \ldots, x_{m}, 0\right) .
$$

On définit $V_{\infty}$ comme étant la limite inductive des $V_{m}$ suivant ces applications.

Donc $V_{\infty}$ est isomorphe à $\mathbb{F}_{2}^{(\mathbb{N})}$, l'espace des suites infinies d'éléments de $\mathbb{F}_{2}$ presque tous nuls.

3.1. L'espace $\mathcal{B}$ des fonctions booléennes. On définit $\mathcal{B}_{m}$ comme étant l'ensemble des fonctions de $V_{m}$ dans $\{ \pm 1\}$. Un élément de $\mathcal{B}_{m}$ est (l'exponentielle d') une fonction booléenne sur $\mathbb{F}_{2}^{m}$ : si $f$ et $g$ sont dans $\mathcal{B}_{m}$, $f g \in \mathcal{B}_{m}$.

On définit de manière duale aux $\phi_{m}$ des applications de transition

$$
\mathcal{B}_{m+1} \rightarrow \mathcal{B}_{m},\left.\quad f \mapsto f\right|_{V_{m}}
$$


où $\left.f\right|_{V_{m}}$ est la restriction de $f$ à $V_{m}$,

$$
\left.f\right|_{V_{m}}:\left(x_{1}, \ldots, x_{m}\right) \mapsto f\left(x_{1}, \ldots, x_{m}, 0\right) .
$$

Cette application permet de définir la limite projective (cf. [2, chap. 1, p. 113])

$$
\mathcal{B}=\mathcal{B}_{\infty}=\operatorname{limproj}_{m \rightarrow \infty} \mathcal{B}_{m}
$$

qui est donc l'ensemble des applications de $V_{\infty}$ à valeurs dans $\{ \pm 1\}$, et les applications $\pi_{m}: \mathcal{B}_{\infty} \rightarrow \mathcal{B}_{m},\left.f \mapsto f\right|_{V_{m}}$.

On munit cet espace d'une structure de groupe topologique telle que les $\pi_{m}^{-1}(\mathbf{1})$ forment un système fondamental de voisinages de l'origine, où $\mathbf{1}$ est la fonction donnant à tous les points de $V_{m}$ l'image 1 . Il est alors compact.

3.2. L'espace de probabilité $\mathcal{B}$. On peut munir l'espace $\mathcal{B}$ d'une structure de probabilité.

On définit une tribu $\mathcal{A}_{m}$ sur $\mathcal{B}_{m}$ en prenant pour $\mathcal{A}_{m}$ l'ensemble des parties $\mathcal{P}\left(\mathcal{B}_{m}\right)$ de $\mathcal{B}_{m}$. L'espace $\mathcal{B}_{m}$ est muni de la probabilité uniforme.

On définit la tribu $\mathcal{A}$ sur $\mathcal{B}$ en prenant pour $\mathcal{A}$ la $\sigma$-algèbre engendrée par $\bigcup \pi_{m}^{-1}\left(\mathcal{A}_{m}\right)$, où l'on a noté $\pi_{m}^{-1}\left(\mathcal{A}_{m}\right)$ l'ensemble des images réciproques des parties de $\mathcal{B}_{m}$ par $\pi_{m}$. On peut définir une probabilité sur cet espace $\mathcal{B}$. Pour chaque $f \in \mathcal{B}_{m}$, la probabilité de l'événement $\pi_{m}^{-1} f$ est donnée par $\underline{\mathrm{P}}\left(\pi_{m}^{-1} f\right)=1 / 2^{q}$ où $q=\left|V_{m}\right|=2^{m}$.

On notera $\mathcal{E}(X)$ l'espérance d'une variable aléatoire $X$ sur $\mathcal{B}$ ou sur $\mathcal{B}_{m}$ :

$$
\mathcal{E}(X)=\int_{\mathcal{B}} X d \underline{\mathrm{P}}
$$

3.3. Transformation de Fourier. Notons $\widehat{V}_{m}$ (resp. $\widehat{V}_{\infty}$ ) l'ensemble des caractères de $V_{m}$ (resp. $V_{\infty}$ ). Le groupe $V_{\infty}$, muni de la topologie discrète, est en dualité avec le groupe $\widehat{V}_{\infty}$ qui est compact et totalement discontinu (cf. [3] auquel on se référera dans ce paragraphe).

3.3.1. Transformation de Fourier sur $V_{m}$. La transformation de Fourier est définie sur les fonctions sur $V_{m}$ à valeurs complexes : à une fonction $f$ de $V_{m}$ dans $\mathbb{C}$, elle fait correspondre une fonction notée $\widehat{f}$ ou $f^{\wedge}$ de $\widehat{V}_{m}$ dans $\mathbb{C}$ par

$$
\widehat{f}(\chi)=\sum_{x \in V_{m}} f(x) \chi(x)
$$

si $\chi$ est dans $\widehat{V}_{m}$.

3.3.2. Transformation de Fourier sur $V_{\infty}$. La transformation de Fourier se prolonge aux fonctions sur le groupe $V_{\infty}$ à valeurs complexes et transforme ces fonctions en distributions à valeurs complexes sur le groupe dual $\widehat{V}_{\infty}$. 
Plus précisément, une distribution sur l'espace $\widehat{V}_{\infty}$ est une forme linéaire sur l'espace des fonctions complexes localement constantes sur $\widehat{V}_{\infty}$ (cf. Bruhat [4]). Si $\varrho$ est une fonction test, c'est-à-dire une fonction sur $V_{\infty}$ nulle en dehors d'un nombre fini de points, on a

$$
\sum_{x \in V_{\infty}} f(x) \varrho(x)=\int_{\widehat{V}_{\infty}} \widehat{f}(\chi) \widehat{\varrho}(\chi) d \chi
$$

où $d \chi$ est la mesure de Haar de $\widehat{V}_{\infty}$, de masse 1. L'expression précédente a un sens si l'on remplace, comme on peut le faire, $V_{\infty}\left(\right.$ et $\left.\widehat{V}_{\infty}\right)$ par $V_{m}$ (et $\left.\widehat{V}_{m}\right)$ pour $m$ assez grand.

Si dans l'équation (3), $f$ est à support dans $V_{m}$ et si $\varrho$ est égale à $\chi$ sur $V_{m}$ et est nulle ailleurs, on retrouve l'équation (2).

\section{4. ÉTUDE DE $\|\widehat{f}\|_{\infty}$}

La relation de Parseval donne, avec $q=2^{m}$,

$$
q=\sum_{x \in V_{m}} f(x)^{2}=\int \widehat{f}(\chi)^{2} d \chi \leq\|\widehat{f}\|_{\infty}^{2}
$$

donc $\|\widehat{f}\|_{\infty}$ est supérieur à $\sqrt{q}$. Il est au plus égal à $q$ car

$$
|\widehat{f}(\chi)|=\left|\sum_{x \in V_{m}} f(x) \chi(x)\right| \leq q .
$$

On va montrer qu'en fait $\|\widehat{f}\|_{\infty}$ est souvent voisin de $\sqrt{q}$. On montre d'abord le lemme suivant.

Lemme 4.1. Si $f$ désigne une fonction de $V_{m}$ à valeurs dans $\{ \pm 1\}, \chi$ un caractère de $V_{m}$, et $\lambda$ un réel, on a

$$
\exp \left(\lambda^{2} q / 2-\lambda^{4} q\right) \leq \mathcal{E}(\exp (\lambda \widehat{f}(\chi))) \leq \exp \left(\lambda^{2} q / 2\right),
$$

où l'espérance est prise sur l'ensemble $\mathcal{B}_{m}$ des fonctions booléennes $f$.

Démonstration. En effet, l'exponentielle s'écrit comme un produit :

$$
\mathcal{E}(\exp (\lambda \widehat{f}(\chi)))=\mathcal{E}\left(\exp \left(\lambda \sum_{x \in V_{m}} f(x) \chi(x)\right)\right)=\mathcal{E}\left(\prod_{x \in V_{m}} \exp (\lambda f(x) \chi(x))\right) .
$$

Écrivons que les variables aléatoires $\exp (\lambda f(x) \chi(x))$ sont indépendantes :

$$
\mathcal{E}\left(\prod_{x \in V_{m}} \exp (\lambda f(x) \chi(x))\right)=\prod_{x \in V_{m}} \mathcal{E}(\exp (\lambda f(x) \chi(x))) .
$$

On vérifie que, pour $x$ fixé, on a

$$
\mathcal{E}(\exp (\lambda f(x) \chi(x)))=\cosh \lambda .
$$


Comme $1+u>\exp \left(u-\frac{1}{2} u^{2}\right)$ si $u>0$ on a

$$
\exp \left(\lambda^{2} / 2-\lambda^{4} / 8\right) \leq 1+\lambda^{2} / 2 \leq \cosh \lambda \leq \exp \left(\lambda^{2} / 2\right),
$$

d'où

$$
\begin{aligned}
\exp \left(\lambda^{2} q / 2-\lambda^{4} q\right) & \leq \exp \left(\lambda^{2} q / 2-\lambda^{4} q / 8\right)=\left(\exp \left(\lambda^{2} / 2-\lambda^{4} / 8\right)\right)^{q} \\
& \leq \mathcal{E}(\exp (\lambda \widehat{f}(\chi))) \leq \exp \left(\lambda^{2} q / 2\right)
\end{aligned}
$$

4.1. Majoration de $\|\widehat{f}\|_{\infty}$. Une variante du théorème 1 , p. 68 , du livre [17] de Kahane donne le résultat suivant, où log désigne le logarithme naturel.

ThÉORÈme 4.1. Si $f$ est une fonction de $V_{m}$ dans $\{ \pm 1\}$, et $\kappa$ un réel plus grand que 2 , on a

$$
\underline{\mathrm{P}}\left(\|\widehat{f}\|_{\infty} \geq(2 q \log (\kappa q))^{1 / 2}\right) \leq 2 / \kappa .
$$

REMARque 4.1. Le résultat est un peu meilleur que celui - général donné par Kahane. Dans notre cas, nous pouvons remarquer que $\|\widehat{f}\|_{\infty}=$ $\widehat{f}(\chi)$ ou $-\widehat{f}(\chi)$ pour au moins une valeur de $\chi$, c'est-à-dire sur un espace de mesure $1 / q$. Donc

$$
\exp \left(\lambda\|\widehat{f}\|_{\infty}\right) \leq \exp (\lambda \widehat{f}(\chi))+\exp (-\lambda \widehat{f}(\chi))
$$

sur un sous-espace de $\widehat{V}_{m}$ de mesure au moins $1 / q$ et par conséquent

$$
\mathcal{E}\left(\exp \left(\lambda\|\widehat{f}\|_{\infty}\right)\right) \leq 2 q \exp \left(q \lambda^{2} / 2\right) .
$$

COROLlaire 4.1. On a presque sûrement

$$
\limsup _{m \rightarrow \infty} \frac{\left\|\left(\pi_{m}(f)\right)^{\wedge}\right\|_{\infty}}{2^{m / 2} \sqrt{m}} \leq \sqrt{2 \log 2}
$$

où $f$ décrit l'espace $\mathcal{B}$ et $\pi_{m}(f)$ désigne la restriction de $f$ à $V_{m}$.

Démonstration. On rappelle que $q=2^{m}$. Prenons $\kappa=2^{m \eta}$ avec $\eta>0$ dans le théorème précédent. On obtient

$$
\underline{\mathrm{P}}\left(\left\|\left(\pi_{m} f\right)^{\wedge}\right\|_{\infty} \geq\left(2^{m+1} m(\eta+1) \log 2\right)^{1 / 2}\right) \leq \frac{2}{2^{m \eta}} .
$$

La somme pour $m \in \mathbb{N}$ est donc convergente :

$$
\sum_{m \in \mathbb{N}} \underline{\mathrm{P}}\left(\left\|\left(\pi_{m} f\right)^{\wedge}\right\|_{\infty} \geq\left(2^{m+1} m(\eta+1) \log 2\right)^{1 / 2}\right)<\infty .
$$

D'après le lemme de Borel-Cantelli (cf. Kahane [17, §1.6], par exemple), on en déduit que, presque sûrement, pour $m$ assez grand on a

$$
\left\|\left(\pi_{m} f\right)^{\wedge}\right\|_{\infty}<\left(2^{m+1} m(\eta+1) \log 2\right)^{1 / 2} .
$$


Cette assertion étant valable pour tout $\eta$ plus grand que 0 , on peut faire tendre $\eta$ vers 0 et on a presque sûrement pour $m$ assez grand

$$
\left\|\left(\pi_{m} f\right)^{\wedge}\right\|_{\infty} \leq\left(2^{m+1} m \log 2\right)^{1 / 2}
$$

donc presque sûrement

$$
\limsup _{m \rightarrow \infty} \frac{\left\|\left(\pi_{m} f\right)^{\wedge}\right\|_{\infty}}{2^{m / 2} \sqrt{m}} \leq \sqrt{2 \log 2} .
$$

Corollaire 4.2. Presque toutes les fonctions booléennes à $m$ variables ont une non-linéarité au moins égale à $2^{m-1}-2^{m / 2-1} \sqrt{2 m \log 2}$.

"Presque toutes" veut dire ici que l'on considère les images par $\pi_{m}$ du complémentaire dans $\mathcal{B}$ d'un ensemble de mesure nulle indépendant de $m$.

Remarque 4.2. Carlet, et d'autre part Olejár et Stanek, obtiennent le résultat du théorème 4.1 à l'aide d'approximations de sommes de coefficients binomiaux $[7,25]$.

4.2. Minoration de $\|\widehat{f}\|_{\infty}$. On aura besoin des lemmes suivants.

LEMME 4.2. Si $f$ désigne une fonction de $V_{m}$ à valeurs dans $\{ \pm 1\}, \chi$ et $\xi$ deux caractères de $V_{m}$, et $\lambda$ un réel, les majorations suivantes sont réalisées :

$$
\mathcal{E}\left(e^{\lambda(\widehat{f}(\chi)+\widehat{f}(\xi))}\right) \leq \begin{cases}e^{\lambda^{2} q} & \text { si } \chi \neq \xi \\ e^{2 \lambda^{2} q} & \text { si } \chi=\xi\end{cases}
$$

Démonstration. La définition de la transformation de Fourier permet d'écrire

$$
\begin{aligned}
\mathcal{E}\left(e^{\lambda(\widehat{f}(\chi)+\widehat{f}(\xi))}\right) & =\mathcal{E}\left(\exp \left(\lambda \sum_{x \in \mathbb{F}_{2}^{m}} f(x)(\chi(x)+\xi(x))\right)\right) \\
& =\mathcal{E}\left(\prod_{x \in \mathbb{F}_{2}^{m}} e^{\lambda f(x)(\chi(x)+\xi(x))}\right)=\prod_{x \in \mathbb{F}_{2}^{m}} \mathcal{E}\left(e^{\lambda f(x)(\chi(x)+\xi(x))}\right)
\end{aligned}
$$

puisque les variables aléatoires $f(x)(\chi(x)+\xi(x))$ sont indépendantes pour $x \in V_{m}$. D'après le lemme 4.1 , le dernier produit est égal à

$$
\begin{aligned}
\prod_{x \in \mathbb{F}_{2}^{m}} \cosh (\lambda f(x)(\chi(x)+\xi(x))) & =\prod_{x \in \mathbb{F}_{2}^{m}} \cosh (\lambda(\chi(x)+\xi(x))) \\
& \leq \prod_{x \in \mathbb{F}_{2}^{m}} e^{\lambda^{2}(\chi(x)+\xi(x))^{2} / 2}
\end{aligned}
$$

d'après la relation (4). Ce dernier terme est égal à

$$
\prod_{x \in \mathbb{F}_{2}^{m}} e^{\lambda^{2}(1+\chi \xi(x))}=\prod_{x \in \mathbb{F}_{2}^{m}} e^{\lambda^{2}} e^{\lambda^{2} \chi \xi(x)}=e^{\lambda^{2} q} \prod_{x \in \mathbb{F}_{2}^{m}} e^{\lambda^{2} \chi \xi(x)}
$$




$$
=e^{\lambda^{2} q} \exp \left(\lambda^{2} \sum_{x \in \mathbb{F}_{2}^{m}} \chi \xi(x)\right)= \begin{cases}e^{\lambda^{2} q} & \text { si } \chi \neq \xi \\ e^{2 \lambda^{2} q} & \text { si } \chi=\xi\end{cases}
$$

en utilisant l'annulation de la somme des valeurs des caractères non triviaux.

On aura également besoin d'une inégalité élémentaire :

LEMME 4.3. Si $X$ est une variable aléatoire de carré intégrable et si $0<\lambda<1$, on $a$

$$
\underline{\mathrm{P}}(X \geq \lambda \mathcal{E}(X)) \geq(1-\lambda)^{2} \frac{\mathcal{E}^{2}(X)}{\mathcal{E}\left(X^{2}\right)} .
$$

Démonstration. Voir par exemple Kahane [17, §1.6].

Le théorème suivant donne une minoration de la probabilité que $\|\widehat{f}\|_{\infty}$ soit assez grand. Il est inspiré de Salem et Zygmund [27] qui traitent le cas du tore. Voir aussi l'article de B. Kashin et L. Tzafriri [18].

THÉORÈme 4.2. Si $f$ désigne une fonction de $V_{m}$ à valeurs dans $\{ \pm 1\}$, et si $0<\alpha<1$ et $0<\eta<1-\alpha^{2}$, alors il existe une constante $B$ positive et ne dépendant que de $\alpha$ et $\eta$ telle que

$$
\underline{\mathrm{P}}\left(\|\widehat{f}\|_{\infty}>\left(\frac{\alpha}{2}-\frac{\eta}{\alpha}-\alpha^{3} \frac{\log q}{q}\right) \sqrt{q \log q}\right)>1-\frac{B}{q^{\eta}} .
$$

Démonstration. Définissons la variable aléatoire $I_{q}=\int_{\widehat{V}_{m}} \exp (\lambda \widehat{f}(\chi)) d \chi$. Le lemme 4.1 permet de minorer l'espérance $\mathcal{E}\left(I_{q}\right)$ de $I_{q}$ sur les fonctions booléennes dans $\mathcal{B}_{m}$ :

$$
\begin{aligned}
\mathcal{E}\left(I_{q}\right) & =\int_{\widehat{V}_{m}} \mathcal{E}(\exp (\lambda \widehat{f}(\chi))) d \chi \\
& \geq \int_{\widehat{V}_{m}} \exp \left(\frac{\lambda^{2} q}{2}-\lambda^{4} q\right) d \chi=\exp \left(\frac{\lambda^{2} q}{2}-\lambda^{4} q\right) .
\end{aligned}
$$

De plus

$$
\begin{aligned}
I_{q}(\chi)^{2} & =\int_{\widehat{V}_{m}} \exp (\lambda \widehat{f}(\chi)) d \chi \int_{\widehat{V}_{m}} \exp (\lambda \widehat{f}(\xi)) d \xi \\
& =\int_{\widehat{V}_{m} \times \widehat{V}_{m}} \exp (\lambda(\widehat{f}(\chi)+\widehat{f}(\xi))) d \chi d \xi
\end{aligned}
$$

d'où, d'après le lemme 4.2,

$$
\begin{aligned}
\mathcal{E}\left(I_{q}(\chi)^{2}\right) & =\int_{\chi, \xi} \mathcal{E}(\exp (\lambda(\widehat{f}(\chi)+\widehat{f}(\xi)))) \\
& \leq \int_{\chi, \xi} \exp \left(q \lambda^{2}\right)+\frac{1}{q} \int_{\chi} \exp \left(2 q \lambda^{2}\right)=\left(1+\frac{\exp \left(q \lambda^{2}\right)}{q}\right) \exp \left(q \lambda^{2}\right) .
\end{aligned}
$$


Donc, d'après l'inégalité du lemme 4.3, si $\eta$ est un réel positif

$$
\begin{aligned}
\underline{\mathrm{P}}\left(I_{q}>q^{-\eta} \exp \left(\frac{\lambda^{2} q}{2}-\lambda^{4} q\right)\right) & \geq\left(1-q^{-\eta}\right)^{2} \frac{\exp \left(\lambda^{2} q-2 \lambda^{4} q\right)}{\left(1+q^{-1} \exp \left(q \lambda^{2}\right)\right) \exp \left(q \lambda^{2}\right)} \\
& \geq\left(1-2 q^{-\eta}\right) \exp \left(-2 \lambda^{4} q\right)\left(1-q^{-1} \exp \left(q \lambda^{2}\right)\right)
\end{aligned}
$$

si $q^{-1} \exp \left(q \lambda^{2}\right)<1$. Cette condition est satisfaite si on choisit

$$
\lambda=\alpha\left(\frac{\log q}{q}\right)^{1 / 2}
$$

avec $0<\alpha<1$. Ce choix de $\lambda$ permet de calculer $\exp \left(-2 \lambda^{4} q\right)$ :

$$
2 \lambda^{4} q=2 \alpha^{4}\left(\frac{\log q}{q}\right)^{2} q=2 \alpha^{4} \frac{(\log q)^{2}}{q}<2 \frac{(\log q)^{2}}{q}
$$

d'où

$$
\begin{aligned}
\underline{\mathrm{P}}\left(I_{q}>q^{-\eta} \exp \left(\frac{\lambda^{2} q}{2}-\lambda^{4} q\right)\right) & \geq\left(1-\frac{2}{q^{\eta}}\right)\left(1-2 \frac{(\log q)^{2}}{q}\right)\left(1-q^{\alpha^{2}-1}\right) \\
& >\left(1-\frac{B}{q^{\eta}}\right)
\end{aligned}
$$

pour une certaine constante $B$ si $\eta<1-\alpha^{2}$.

Il est évident que

$$
\exp \left(\lambda\|\widehat{f}\|_{\infty}\right) \geq \int_{\widehat{V}_{m}} \exp (\lambda \widehat{f}(\chi))
$$

D'où

$\underline{\mathrm{P}}\left(\exp \left(\lambda\|\widehat{f}\|_{\infty}\right)>q^{-\eta} \exp \left(\frac{\lambda^{2} q}{2}-\lambda^{4} q\right)\right) \geq \underline{\mathrm{P}}\left(I_{q}>q^{-\eta} \exp \left(\frac{\lambda^{2} q}{2}-\lambda^{4} q\right)\right)$.

L'événement du membre de gauche peut encore s'écrire

ou encore

$$
\|\widehat{f}\|_{\infty}>\frac{\lambda q}{2}-\lambda^{3} q-\frac{\eta \log q}{\lambda}
$$

$$
\|\widehat{f}\|_{\infty}>\frac{\alpha}{2} \sqrt{q \log q}-\lambda^{3} q-\frac{\eta \log q}{\lambda} .
$$

Majorons le deuxième terme de cette somme :

$$
\lambda^{3} q=\alpha^{3}\left(\frac{\log q}{q}\right)^{3 / 2} q=\alpha^{3} \frac{(\log q)^{3 / 2}}{q^{1 / 2}}=\alpha^{3} \frac{\log q}{q} \sqrt{q \log q} .
$$

Enfin le troisième terme vaut

$$
\frac{\eta \log q}{\lambda}=\frac{\eta \log q}{\alpha}\left(\frac{q}{\log q}\right)^{1 / 2}=\frac{\eta}{\alpha} \sqrt{q \log q} .
$$


L'événement en question s'écrit donc

$$
\|\widehat{f}\|_{\infty}>\left(\frac{\alpha}{2}-\frac{\eta}{\alpha}-\alpha^{3} \frac{\log q}{q}\right) \sqrt{q \log q},
$$

ce qui termine la démonstration.

Corollaire 4.3. On a presque sûrement

$$
\liminf _{m \rightarrow \infty} \frac{\left\|\left(\pi_{m}(f)\right)^{\wedge}\right\|_{\infty}}{2^{m / 2} \sqrt{m}} \geq \frac{\sqrt{\log 2}}{2}
$$

où $f$ décrit les éléments de l'espace $\mathcal{B}$ et $\pi_{m}(f)$ désigne la restriction de $f$ $\grave{a} V_{m}$.

Démonstration. En effet, en faisant la somme pour $m \in \mathbb{N}$ des inégalités données par le théorème précédent, on obtient, en remarquant que $q=2^{m}$,

$$
\begin{aligned}
\sum_{m} \underline{\mathrm{P}}\left(\left\|\left(\pi_{m}(f)\right)^{\wedge}\right\|_{\infty}<\left(\frac{\alpha}{2}-\frac{\eta}{\alpha}-\alpha^{3} \frac{m \log 2}{2^{m}}\right) 2^{m / 2}\right. & \sqrt{m \log 2}) \\
& <\sum_{m} \frac{B}{2^{m \eta}}<\infty .
\end{aligned}
$$

Donc, le lemme de Borel-Cantelli nous dit que p.s.

$$
\left\|\left(\pi_{m}(f)\right)^{\wedge}\right\|_{\infty}>\left(\frac{\alpha}{2}-\frac{\eta}{\alpha}-\alpha^{3} \frac{m \log 2}{2^{m}}\right) 2^{m / 2} \sqrt{m \log 2}
$$

sauf pour un nombre fini de $m$, c'est-à-dire p.s.

$$
\liminf _{m \rightarrow \infty} \frac{\left\|\left(\pi_{m}(f)\right)^{\wedge}\right\|_{\infty}}{2^{m / 2} \sqrt{m}} \geq\left(\frac{\alpha}{2}-\frac{\eta}{\alpha}\right) \sqrt{\log 2}
$$

On peut faire tendre $\alpha$ vers 1 et $\eta$ vers 0 . On obtient

$$
\liminf _{m \rightarrow \infty} \frac{\left\|\left(\pi_{m}(f)\right)^{\wedge}\right\|_{\infty}}{2^{m / 2} \sqrt{m}} \geq \frac{\sqrt{\log 2}}{2} \quad \text { p.s. }
$$

Corollaire 4.4. Presque toutes les fonctions booléennes à $m$ variables ont une non-linéarité au plus égale à $2^{m-1}-2^{m / 2-2} \sqrt{m \log 2}$.

\section{5. ÉTUDE DE $\|\widehat{f}\|_{4}$}

Reprenons l'idée de D. Newman et J. Byrnes [23]. Ils ont remarqué que, dans le cas des séries de Fourier sur $\mathbb{Z}$, la norme dans $L^{4}$ de $\sum_{n} \pm e^{i n t}$ avait une expression agréable. Il en va de même de $\|\widehat{f}\|_{4}$ pour $f: V_{m} \rightarrow\{ \pm 1\}$. On remarque que

$$
\|\widehat{f}\|_{2} \leq\|\widehat{f}\|_{4} \leq\|\widehat{f}\|_{\infty} .
$$

En effet, la première inégalité vient de ce que les fonction $\widehat{f}$ sont définies sur un espace de mesure égale à 1 . Par conséquent, la conjecture 2.1 implique une conjecture plus faible : 
Conjecture 5.1. Si $f$ décrit l'espace des fonctions de $V_{m}$ dans $\{ \pm 1\}$, on $a$

$$
\lim _{m \rightarrow \infty} \inf _{f \in V_{m}} \frac{\|\widehat{f}\|_{4}}{2^{m / 2}}=1
$$

Cela a également été étudié par X. M. Zhang et Y. L. Zheng [29] ou par P. Stănică [28] sous le nom de "somme des carrés". On peut voir également l'article de P. Langevin et P. Solé [20] qui appliquent cette notion à une cubique.

5.1. L'expression de $\|\widehat{f}\|_{4}$. On obtient l'expression simple suivante pour $\|\widehat{f}\|_{4}$.

Lemme 5.1. Si $f$ est une fonction de $V_{m}$ à valeurs dans $\{ \pm 1\}$,

$$
\|\widehat{f}\|_{4}^{4}=\sum_{x_{1}+x_{2}+x_{3}+x_{4}=0} f\left(x_{1}\right) f\left(x_{2}\right) f\left(x_{3}\right) f\left(x_{4}\right) .
$$

Démonstration. Décomposons $\widehat{f}^{4}$ et inversons l'ordre de la somme et de l'intégrale :

$$
\begin{aligned}
\|\widehat{f}\|_{4}^{4}= & \int_{\widehat{V}_{m}} \widehat{f}^{4} d \chi \\
= & \int_{\widehat{V}_{m}}\left(\sum_{V_{m}} f\left(x_{1}\right) \chi\left(x_{1}\right)\right)\left(\sum_{V_{m}} f\left(x_{2}\right) \chi\left(x_{2}\right)\right) \\
& \times\left(\sum_{V_{m}} f\left(x_{3}\right) \chi\left(x_{3}\right)\right)\left(\sum_{V_{m}} f\left(x_{4}\right) \chi\left(x_{4}\right)\right) d \chi \\
= & \sum_{x_{1}, x_{2}, x_{3}, x_{4}} f\left(x_{1}\right) f\left(x_{2}\right) f\left(x_{3}\right) f\left(x_{4}\right) \int_{\widehat{V}_{m}} \chi\left(x_{1}+x_{2}+x_{3}+x_{4}\right) d \chi \\
= & \sum_{x_{1}+x_{2}+x_{3}+x_{4}=0} f\left(x_{1}\right) f\left(x_{2}\right) f\left(x_{3}\right) f\left(x_{4}\right) .
\end{aligned}
$$

5.1.1. Réécriture de la conjecture 2.1. Décomposons la somme donnée dans le lemme 5.1 :

$$
\begin{aligned}
\sum_{x_{1}+x_{2}+x_{3}+x_{4}=0} f\left(x_{1}\right) & f\left(x_{2}\right) f\left(x_{3}\right) f\left(x_{4}\right) \\
& =q^{2}+\sum_{a \neq 0} \sum_{x_{1}+x_{2}=x_{3}+x_{4}=a} f\left(x_{1}\right) f\left(x_{2}\right) f\left(x_{3}\right) f\left(x_{4}\right)
\end{aligned}
$$

et on vérifie

$$
\begin{aligned}
& \sum_{x_{1}+x_{2}=x_{3}+x_{4}=a} f\left(x_{1}\right) f\left(x_{2}\right) f\left(x_{3}\right) f\left(x_{4}\right) \\
= & \sum_{x_{1}+x_{2}=a} f\left(x_{1}\right) f\left(x_{2}\right) \sum_{x_{3}+x_{4}=a} f\left(x_{3}\right) f\left(x_{4}\right)=\left(\sum_{x_{1}+x_{2}=a} f\left(x_{1}\right) f\left(x_{2}\right)\right)^{2} \geq 0 .
\end{aligned}
$$


Définissons les variables aléatoires à valeurs dans $\mathbb{C}$ et dépendant de $a$ dans $V_{m}$ :

$$
X_{a}=\left(\sum_{x_{1}+x_{2}=a} f\left(x_{1}\right) f\left(x_{2}\right)\right)^{2},
$$

d'où

$$
\|\widehat{f}\|_{4}^{4}-q^{2}=\sum_{a \neq 0, a \in V_{m}} X_{a} .
$$

La conjecture 2.1 se réécrit de la manière suivante.

Conjecture 5.2. Pour tout $\varepsilon>0$, il existe $q$ non carré et $f$ dans $V_{m}$ tels que $\sum_{a \neq 0} X_{a}<\varepsilon q^{2}$ où $X_{a}$ est donné par la formule (6).

On comparera avec le problème 14.3 dans l'article de T. Erdélyi [14].

5.2. Calculs d'espérances. Remarquons que $\|\widehat{f}\|_{4}^{4}$ est compris entre $q^{2}$ et $q^{3}$. En effet, la première inégalité $\|\widehat{f}\|_{4}^{4} \geq q^{2}$ vient de l'inéquation (5). Le lemme 5.1 implique d'autre part que $\|\widehat{f}\|_{4}^{4} \leq q^{3}$. Voir [29, théorème 6$]$ ou $[6$, théorème 1$]$.

On peut également déduire du lemme 5.1 le calcul des espérances $\mathcal{E}\left(\|\widehat{f}\|_{4}^{4}\right)$ et de $\mathcal{E}\left(\|\widehat{f}\|_{4}^{8}\right)$ relativement à l'ensemble $\mathcal{B}_{m}$ des fonctions booléennes $f$. On utilise pour cela les lemmes suivants. Remarquons d'abord que

$$
\mathcal{E}\left(\|\widehat{f}\|_{4}^{4}\right)=\sum_{x_{1}+x_{2}+x_{3}+x_{4}=0} \mathcal{E}\left(f\left(x_{1}\right) f\left(x_{2}\right) f\left(x_{3}\right) f\left(x_{4}\right)\right) .
$$

LEMME 5.2. On a

$$
\mathcal{E}\left(f\left(x_{1}\right) f\left(x_{2}\right) \cdots f\left(x_{r}\right)\right)=\mathcal{E}\left(f\left(x_{3}\right) f\left(x_{4}\right) \cdots f\left(x_{r}\right)\right)
$$

si $x_{1}=x_{2}$.

Démonstration. Si $x_{1}=x_{2}$, on a

$$
f\left(x_{1}\right) f\left(x_{2}\right) \cdots f\left(x_{r}\right)=f\left(x_{3}\right) f\left(x_{4}\right) \cdots f\left(x_{r}\right) .
$$

LEMME 5.3. On a

$$
\mathcal{E}\left(f\left(x_{1}\right) f\left(x_{2}\right) f\left(x_{3}\right) \cdots f\left(x_{r}\right)\right)=0 \text { ou } 1 .
$$

L'espérance $\mathcal{E}\left(f\left(x_{1}\right) f\left(x_{2}\right) f\left(x_{3}\right) \cdots f\left(x_{r}\right)\right)$ est égale à 1 si et seulement si pour chaque $y \in \mathbb{F}_{2}^{m}$ l'ensemble des $x_{i}$ égaux à $y$ a un cardinal pair, c'est$\grave{a}$-dire si et seulement si il existe une partition de $\left\{x_{1}, x_{2}, x_{3}, \ldots, x_{r}\right\}$ en couples formés d'éléments égaux.

Démonstration. L'application successive du lemme précédent permet de se ramener au cas où tous les $x_{i}$ sont distincts. Les variables aléatoires $f\left(x_{i}\right)$ sont alors indépendantes, donc

$$
\mathcal{E}\left(f\left(x_{1}\right) f\left(x_{2}\right) f\left(x_{3}\right) \cdots f\left(x_{r}\right)\right)=\mathcal{E}\left(f\left(x_{1}\right)\right) \mathcal{E}\left(f\left(x_{2}\right)\right) \mathcal{E}\left(f\left(x_{3}\right)\right) \cdots \mathcal{E}\left(f\left(x_{r}\right)\right) .
$$


De plus $f\left(x_{1}\right)=1$ ou -1 avec la probabilité $1 / 2$, d'où $\mathcal{E}\left(f\left(x_{1}\right)\right)=0$. Donc si tous les $x_{i}$ sont distincts, $\mathcal{E}\left(f\left(x_{1}\right) f\left(x_{2}\right) f\left(x_{3}\right) \cdots f\left(x_{r}\right)\right)=0$ sauf si la suite des $x_{i}$ est vide auquel cas elle vaut $\mathcal{E}(1)=1$.

Posons

$$
E\left(a_{1}, \ldots, a_{r}\right)=\sum_{x_{1}, \ldots, x_{r}} \mathcal{E}\left(f\left(x_{1}\right) f\left(x_{1}+a_{1}\right) \cdots f\left(x_{r}\right) f\left(x_{r}+a_{r}\right)\right) .
$$

Lemme 5.4. Si a et les $a_{i}$ sont dans $V_{m}$ et si $a \neq 0$, on a

$$
E\left(a, a_{1}, \ldots, a_{r}\right) \leq 2 \sum_{1 \leq i \leq r} E\left(a_{1}, \ldots, a_{i}+a, \ldots, a_{r}\right)
$$

Démonstration. Appliquons le lemme 5.3 :

$$
\begin{aligned}
& E\left(a, a_{1}, \ldots, a_{r}\right) \\
& =\sum_{x, x_{1}, \ldots, x_{r}} \mathcal{E}\left(f(x) f(x+a) f\left(x_{1}\right) f\left(x_{1}+a_{1}\right) \cdots f\left(x_{r}\right) f\left(x_{r}+a_{r}\right)\right) \\
& =\sum_{x_{1}, x_{2}, \ldots, x_{r}} \sum \mathcal{E}\left(f(x) f(x+a) f\left(x_{1}\right) f\left(x_{1}+a_{1}\right) \cdots f\left(x_{r}\right) f\left(x_{r}+a_{r}\right)\right)
\end{aligned}
$$

où la dernière somme est sur les $x$ dans $\left\{x_{1}, x_{1}+a_{1}, \ldots, x_{r}, x_{r}+a_{r}\right\}$. Si $x=x_{1}$, le lemme 5.2 permet d'écrire

$$
\begin{aligned}
\mathcal{E}(f(x) f(x+ & \left.a) f\left(x_{1}\right) f\left(x_{1}+a_{1}\right) \cdots f\left(x_{r}\right) f\left(x_{r}+a_{r}\right)\right) \\
& =\mathcal{E}\left(f\left(x_{1}\right) f\left(x_{1}+a\right) f\left(x_{1}\right) f\left(x_{1}+a_{1}\right) \cdots f\left(x_{r}\right) f\left(x_{r}+a_{r}\right)\right) \\
& =\mathcal{E}\left(f\left(x_{1}+a\right) f\left(x_{1}+a_{1}\right) \cdots f\left(x_{r}\right) f\left(x_{r}+a_{r}\right)\right) \\
& =\mathcal{E}\left(f(t) f\left(t+a_{1}+a\right) \cdots f\left(x_{r}\right) f\left(x_{r}+a_{r}\right)\right)
\end{aligned}
$$

en posant $t=x_{1}+a_{1}$. Si $x=x_{1}+a_{1}$, on a de même

$$
\begin{aligned}
\mathcal{E}(f(x) & \left.f(x+a) f\left(x_{1}\right) f\left(x_{1}+a_{1}\right) \cdots f\left(x_{r}\right) f\left(x_{r}+a_{r}\right)\right) \\
& =\mathcal{E}\left(f\left(x_{1}\right) f\left(x_{1}+a+a_{1}\right) f\left(x_{2}\right) f\left(x_{2}+a_{2}\right) \cdots f\left(x_{r}\right) f\left(x_{r}+a_{r}\right)\right)
\end{aligned}
$$

d'où le lemme.

5.2.1. Les espérances de $X_{a}, X_{a}^{2}, X_{a} X_{b}$ sur $\mathcal{B}_{m}$

Proposition 5.1. Si a est un élément non nul de $V_{m}$, on a $\mathcal{E}\left(X_{a}\right)$ $=2 q$.

Démonstration. Décomposons $\mathcal{E}\left(X_{a}\right)$ :

$$
\begin{aligned}
\mathcal{E}\left(\sum_{x_{1}+x_{2}=a} f\left(x_{1}\right) f\left(x_{2}\right)\right)^{2} & =\mathcal{E}\left(\sum_{x_{1} \in V_{m}} f\left(x_{1}\right) f\left(x_{1}+a\right)\right)^{2} \\
& =\sum_{x_{1}, x_{2}} \mathcal{E}\left(f\left(x_{1}\right) f\left(x_{1}+a\right) f\left(x_{2}\right) f\left(x_{2}+a\right)\right) .
\end{aligned}
$$


Les valeurs de $\left(x_{1}, x_{2}\right)$ qui rendent $\mathcal{E}\left(f\left(x_{1}\right) f\left(x_{1}+a\right) f\left(x_{2}\right) f\left(x_{2}+a\right)\right)$ égale à 1 sont $x_{1}=x_{2}$, et $x_{1}=x_{2}+a$. Il y en a $q$ dans les deux cas. Ceci entraîne la proposition.

Proposition 5.2. Si a est un élément non nul de $V_{m}$, on a $\mathcal{E}\left(X_{a}^{2}\right)$ $\leq 12 q^{2}$.

Démonstration. Décomposons $X_{a} X_{a}$ :

$$
\begin{aligned}
X_{a} X_{a}= & \left(\sum_{x} f(x) f(x+a)\right)^{2}\left(\sum_{x} f(x) f(x+a)\right)^{2} \\
= & \left(\sum_{x} f(x) f(x+a)\right)\left(\sum_{y} f(y) f(y+a)\right) \\
& \times\left(\sum_{z} f(z) f(z+a)\right)\left(\sum_{t} f(t) f(t+a)\right) \\
= & \sum_{x, y, z, t} f(x) f(x+a) f(y) f(y+a) f(z) f(z+a) f(t) f(t+a),
\end{aligned}
$$

d'où, d'après le lemme 5.4,

$$
\begin{aligned}
\mathcal{E}\left(X_{a}^{2}\right) & =\sum_{x, y, z, t} \mathcal{E}(f(x) f(x+a) f(y) f(y+a) f(z) f(z+a) f(t) f(t+a)) \\
& =E(a, a, a, a) \\
& \leq 2 E(0, a, a)+2 E(a, 0, a)+2 E(a, a, 0)=6 q E(a, a),
\end{aligned}
$$

d'après le lemme 5.2. Ce dernier terme vaut $12 q^{2}$ grâce à la proposition 5.1.

Proposition 5.3. Si a et $b$ sont des éléments non nuls et distincts de $V_{m}$, on a $\mathcal{E}\left(X_{a} X_{b}\right) \leq 4 q^{2}+32 q$.

Démonstration. Décomposons $X_{a} X_{b}$ :

$$
\begin{aligned}
X_{a} X_{b}= & \left(\sum_{x} f(x) f(x+a)\right)^{2}\left(\sum_{x} f(x) f(x+b)\right)^{2} \\
= & \left(\sum_{x} f(x) f(x+a)\right)\left(\sum_{y} f(y) f(y+a)\right) \\
& \times\left(\sum_{z} f(z) f(z+b)\right)\left(\sum_{t} f(t) f(t+b)\right) \\
= & \sum_{x, y, z, t} f(x) f(x+a) f(y) f(y+a) f(z) f(z+b) f(t) f(t+b) .
\end{aligned}
$$

On a donc, d'après de lemme 5.3 ,

$$
\begin{aligned}
\mathcal{E}\left(X_{a} X_{b}\right) & =\sum_{x, y, z, t} \mathcal{E}(f(x) f(x+a) f(y) f(y+a) f(z) f(z+b) f(t) f(t+b)) \\
& =E(a, a, b, b)
\end{aligned}
$$




$$
\begin{aligned}
& \leq 2 E(0, b, b)+2 E(a, b+a, b)+2 E(a, b, b+a) \\
& =2 E(0, b, b)+4 E(a, b+a, b),
\end{aligned}
$$

en utilisant l'égalité $E(a, b+a, b)=E(a, b, b+a)$. Du lemme 5.2 on obtient

$$
\mathcal{E}\left(X_{a} X_{b}\right) \leq 2 q E(b, b)+4 E(a, b+a, b) .
$$

La première somme est calculée dans la proposition 5.1. Calculons la deuxième somme, en utilisant le lemme 5.4 :

$$
E(a, b+a, b) \leq 2 E(b, b)+2 E(b+a, b+a)=8 q
$$

d'après la proposition 5.1 .

5.2.2. Espérances de $\|\widehat{f}\|_{4}^{4}$ et $\|\widehat{f}\|_{4}^{8}$

Proposition 5.4. Si $f$ est une fonction aléatoire de $V_{m}$ dans $\{ \pm 1\}$, alors $\mathcal{E}\left(\|\widehat{f}\|_{4}^{4}\right)=3 q^{2}-2 q$.

Démonstration. En effet

$$
\|\widehat{f}\|_{4}^{4}=q^{2}+\sum_{a \neq 0} X_{a} .
$$

Donc

$$
\mathcal{E}\left(\|\widehat{f}\|_{4}^{4}\right)=q^{2}+\sum_{a \neq 0} \mathcal{E}\left(X_{a}\right)=q^{2}+2 q(q-1) .
$$

Proposition 5.5. Si $f$ est une fonction aléatoire de $V_{m}$ dans $\{ \pm 1\}$, alors $\mathcal{E}\left(\|\widehat{f}\|_{4}^{8}\right) \leq 64 q-100 q^{2}+28 q^{3}+9 q^{4}$.

Démonstration. On a

$$
\|\widehat{f}\|_{4}^{8}=\left(q^{2}+\sum_{a \neq 0} X_{a}\right)^{2}=q^{4}+2 q^{2} \sum_{a \neq 0} X_{a}+\sum_{a \neq 0} X_{a}^{2}+\sum_{0 \neq a \neq b \neq 0} X_{a} X_{b} .
$$

Donc

$$
\begin{aligned}
& \mathcal{E}\left(\|\widehat{f}\|_{4}^{8}\right)=q^{4}+2 q^{2} \sum_{a \neq 0} \mathcal{E}\left(X_{a}\right)+\sum_{a \neq 0} \mathcal{E}\left(X_{a}^{2}\right)+\sum_{0 \neq a \neq b \neq 0} \mathcal{E}\left(X_{a} X_{b}\right) \\
& \leq q^{4}+2 q^{2}(q-1) 2 q+12 q^{2}(q-1)+(q-1)(q-2)\left(4 q^{2}+32 q\right) \\
& =64 q-100 q^{2}+28 q^{3}+9 q^{4} \text {. }
\end{aligned}
$$

\subsection{Inégalités sur $\|\widehat{f}\|_{4}^{4}$}

Proposition 5.6. Si $f$ est une fonction aléatoire de $V_{m}$ dans $\{ \pm 1\}$, et $t$ un réel positif, alors

$$
\underline{\mathrm{P}}\left(\left|\frac{\|\widehat{f}\|_{4}^{4}}{q^{2}}-3+\frac{2}{q}\right| \geq t\right) \leq \frac{40}{t^{2} q} .
$$

Démonstration. La variance de $\|\widehat{f}\|_{4}^{4}$ vérifie, d'après le paragraphe 5.2.2,

$$
\operatorname{var}\left(\|\widehat{f}\|_{4}^{4}\right)=\mathcal{E}\left(\|\widehat{f}\|_{4}^{8}\right)-\mathcal{E}\left(\|\widehat{f}\|_{4}^{4}\right)^{2} \leq 64 q-104 q^{2}+40 q^{3} .
$$


Donc, en appliquant l'inégalité de Bienaymé-Tchebycheff (voir par exemple Kahane $[17, \S 1.6])$,

$$
\underline{\mathrm{P}}\left(\left|\|\widehat{f}\|_{4}^{4}-\mathcal{E}\left(\|f\|_{4}^{4}\right)\right| \geq u\right) \leq \frac{\operatorname{var}\left(\|f\|_{4}^{4}\right)}{u^{2}} \leq \frac{64 q-104 q^{2}+40 q^{3}}{u^{2}}
$$

pour $u>0$. En remplaçant $u$ par $q^{2} t$, on obtient

$$
\underline{\mathrm{P}}\left(\left|\|\widehat{f}\|_{4}^{4}-3 q^{2}+2 q\right| \geq q^{2} t\right) \leq \frac{64 q-104 q^{2}+40 q^{3}}{q^{4} t^{2}} \leq \frac{40}{t^{2} q},
$$

d'où le résultat.

5.4. Étude asymptotique de $\|\widehat{f}\|_{4}$. Pour presque tout $f$ appartenant à $\mathcal{B},\left\|\left(\pi_{m} f\right)^{\wedge}\right\|_{4} / 2^{m / 2}$ a une limite donnée par le corollaire suivant.

Corollaire 5.1. Si $f$ est une fonction aléatoire dans $\mathcal{B}$, on a presque sûrement

$$
\lim _{m} \frac{\left\|\left(\pi_{m} f\right)^{\wedge}\right\|_{4}}{2^{m / 2}}=3^{1 / 4} .
$$

Démonstration. Rappelons que $q=2^{m}$. Faisons la somme pour $m \in \mathbb{N}$ des inégalités données par la proposition précédente :

$$
\sum_{m} \underline{\mathrm{P}}\left(\left|\frac{\left\|\left(\pi_{m} f\right)^{\wedge}\right\|_{4}^{4}}{2^{2 m}}-3+\frac{2}{2^{m}}\right| \geq t\right) \leq \sum_{m} \frac{40}{t^{2} 2^{m}}<\infty .
$$

Par conséquent, le lemme de Borel-Cantelli dit que, pour $t$ donné, on a presque sûrement

$$
\left|\frac{\left\|\left(\pi_{m} f\right)^{\wedge}\right\|_{4}^{4}}{2^{2} m}-3+\frac{2}{2^{m}}\right|<t
$$

sauf peut-être pour un nombre fini de $q$. Par conséquent, on a presque sûrement

$$
\lim _{m} \frac{\left\|\left(\pi_{m} f\right)^{\wedge}\right\|_{4}^{4}}{2^{2 m}}=3
$$

\subsection{Résultats asymptotiques}

5.5.1. Convergence de la loi de la variable aléatoire $q^{-1} X_{a}$. On notera

$$
\Phi_{X}(u)=\mathcal{E}(\exp (i u X))
$$

la fonction caractéristique d'une variable aléatoire $X$.

Proposition 5.7. La distribution de $q^{-1 / 2}\left(\sum_{x \in \mathbb{F}_{2}^{m}} f(x) f(x+a)\right)$ converge en loi vers la distribution gaussienne d'espérance nulle et de variance 2 quand $q$ tend vers l'infini.

Démonstration. Soit $H$ l'hyperplan de l'espace vectoriel $\mathbb{F}_{2}^{m}$ orthogonal à $a$. Les variables aléatoires $f(x) f(x+a)$ sont indépendantes pour $x \in H$ et 
on a

$$
\frac{1}{\sqrt{q}}\left(\sum_{x \in \mathbb{F}_{2}^{m}} f(x) f(x+a)\right)=\frac{2}{\sqrt{q}}\left(\sum_{x \in H} f(x) f(x+a)\right) .
$$

Le développement de Taylor de $\Phi_{f(x) f(x+a)}$ à l'origine est donné par

$$
\begin{aligned}
\Phi_{f(x) f(x+a)}(u)= & 1+i u \mathcal{E}(f(x) f(x+a)) \\
& -u^{2} \mathcal{E}(f(x) f(x+a) f(x) f(x+a)) / 2+o\left(u^{2}\right) \\
= & 1-u^{2} / 2+o\left(u^{2}\right)
\end{aligned}
$$

dans un voisinage de l'origine. Donc

$$
\log \Phi_{f(x) f(x+a)}(u)=-u^{2} / 2+o\left(u^{2}\right)
$$

dans un voisinage $\mathcal{V}$ de l'origine.

Posons

$$
S_{q}=\sum_{x \in H} f(x) f(x+a)
$$

et soit $\Phi_{S_{q} / \sqrt{q}}(u)=\mathcal{E}\left(\exp \left(i u S_{q} / \sqrt{q}\right)\right)$ la fonction caractéristique de $S_{q} / \sqrt{q}$. On a

$$
\Phi_{S_{q} / \sqrt{q}}(u)=\mathcal{E}\left(\exp \left(i u S_{q} / \sqrt{q}\right)\right)=\mathcal{E}\left(\exp \left(i S_{q} u / \sqrt{q}\right)\right)=\Phi_{S_{q}}(u / \sqrt{q})
$$

Écrivons que les variables aléatoires $f(x) f(x+a)$ sont indépendantes pour $x \in H$ :

$$
\Phi_{S_{q}}(u / \sqrt{q})=\prod_{x \in H} \Phi_{f(x) f(x+a)}(u / \sqrt{q})
$$

d'où

$$
\begin{aligned}
\log \Phi_{S_{q}}(u / \sqrt{q}) & =\sum_{x \in H} \log \Phi_{f(x) f(x+a)}(u / \sqrt{q})=\sum_{x \in H}\left(-u^{2} / 2 q+o\left(u^{2} / q\right)\right) \\
& =-u^{2} / 4+q o\left(u^{2} / q\right)
\end{aligned}
$$

dès que $q$ est assez grand pour que $u / \sqrt{q}$ soit dans le voisinage $\mathcal{V}$ de 0 . Par conséquent, quand $q$ tend vers l'infini, la fonction caractéristique $\Phi_{S_{q} / \sqrt{q}}$ tend vers $\exp \left(-u^{2} / 4\right)$, donc vers la fonction caractéristique d'une loi normale centrée, de variance $1 / 2$.

La distribution de $q^{-1 / 2}\left(\sum_{x \in \mathbb{F}_{2}^{m}} f(x) f(x+a)\right)$ égale à $2 S_{q} / \sqrt{q}$ converge donc en loi vers une loi normale centrée, de variance 2.

Posons $Y_{a}=q^{-1} X_{a}$.

Proposition 5.8. La distribution de $Y_{a}=q^{-1} X_{a}$ converge en loi vers la distribution de densité

$$
\frac{1}{2 \sqrt{\pi x}} e^{-x / 4} \mathbf{1}_{(x>0)}
$$


Démonstration. En effet, la variable $Y_{a}$ est égale à

$$
\left(q^{-1 / 2}\left(\sum_{x \in \mathbb{F}_{2}^{m}} f(x) f(x+a)\right)\right)^{2},
$$

et le carré d'une distribution gaussienne d'espérance nulle et de variance 2 est une variable aléatoire de densité

$$
\frac{1}{2 \sqrt{\pi x}} e^{-x / 4} \mathbf{1}_{(x>0)} .
$$

5.5.2. Le théorème de Gärtner-Ellis. Il s'agit, comme le théorème de Cramer, d'un théorème concernant une évaluation des grandes déviations d'une variable aléatoire. On pourra se référer à J. Bucklew [5] ou à A. Dembo et O. Zeitouni [12].

On a

$$
\frac{1}{q^{2}}\|\widehat{f}\|_{4}^{4}-1=\frac{1}{q} \sum_{a \neq 0} Y_{a} .
$$

On a vu que les variables aléatoires $Y_{a}$ avaient presque la même distribution. Si elles étaient indépendantes, on pourrait résoudre la conjecture 2.1 en utilisant le théorème de Cramer, qui donne une évaluation des grandes déviations pour des variables aléatoires indépendantes et équidistribuées.

Mais cela n'est pas le cas ici. Le théorème de Gärtner-Ellis peut peut-être s'appliquer, mais il faut vérifier un certain nombre d'hypothèses. Définissons

$$
\phi_{q}(u)=\frac{1}{q} \log \mathcal{E}\left(\exp \left(u \sum_{a \neq 0} Y_{a}\right)\right) .
$$

Supposons que $\phi(u)=\lim _{q \rightarrow \infty} \phi_{q}(u)$ existe pour tout $u \in \mathbb{R}$ (prenant éventuellement des valeurs infinies) et soit différentiable sur l'ensemble $D_{\phi}=$ $\{u \mid \phi(u)<\infty\}$.

Soit aussi

$$
I(x)=\sup _{u}(u x-\phi(u)) \quad \text { et } \quad \phi^{\prime}\left(D_{\phi}\right)=\left\{\phi^{\prime}(u) \mid u \in D_{\phi}\right\} .
$$

Le théorème de Gärtner-Ellis implique

$$
\limsup _{q \rightarrow \infty} \frac{1}{q} \log \underline{\mathrm{P}}\left(\frac{1}{q} \sum_{a \neq 0} Y_{a}<\varepsilon\right) \leq-\inf _{x<\varepsilon} I(x) .
$$

Comme pour $q$ carré, il existe des fonctions courbes, la probabilité que $\|\widehat{f}\|_{4}^{4}=q^{2}$ est supérieure à $1 / 2^{q}$, donc

$$
-\log 2 \leq-\inf _{x<\varepsilon} I(x)
$$

Mais si $] 0, \varepsilon\left[\subset \phi^{\prime}\left(D_{\phi}\right)\right.$, le théorème de Gärtner-Ellis implique

$$
\liminf _{q \rightarrow \infty} \frac{1}{q} \log \underline{\mathrm{P}}\left(\frac{1}{q} \sum_{a \neq 0} Y_{a}<\varepsilon\right) \geq-\inf _{x<\varepsilon} I(x) .
$$


On en déduirait que pour $\varepsilon$ donné, pour tout $q$ assez grand, il existe $f$ tel que

$$
\frac{1}{q^{2}}\|\widehat{f}\|_{4}^{4}-1<\varepsilon,
$$

donc tel que

$$
\frac{1}{\sqrt{q}}\|\widehat{f}\|_{4}-1<\varepsilon .
$$

\section{Références}

[1] C. Adams and S. Mister, Practical S-box design, preprint, http://adonis.ee. queensu.ca:8000/cast/psbd.ps.

[2] N. Bourbaki, Eléments de mathématiques, Algèbre I, chap. 1 à 3, Hermann, Paris, 1970.

[3] —, Eléments de mathématiques, Théorie spectrale, chap. 1 et 2, Hermann, Paris, 1967.

[4] F. Bruhat, Distributions sur un groupe localement compact et applications à l'étude des représentations des groupes p-adiques, Bull. Soc. Math. France 89 (1961), 43-75.

[5] J. Bucklew, Large deviation techniques in decision, simulation, and estimation, Wiley Ser. Probab. Math. Statist. Appl. Probab. Statist., John Wiley \& Sons, New York, 1990.

[6] A. Canteaut, C. Carlet, P. Charpin and C. Fontaine, Propagation characteristics and correlation-immunity of highly nonlinear Boolean functions, dans : Advances in Cryptology -EUROCRYPT 2000 (Bruges), Lecture Notes in Comput. Sci. 1807, Springer, Berlin, 2000, 507-522.

[7] C. Carlet, On cryptographic complexity of Boolean functions, dans : Proc. Sixth Conf. on Finite Fields with Applications to Coding Theory, Cryptography and Related Areas, G. L. Mullen, H. Stichtenoth and H. Tapia-Recillas (eds.), Springer, Berlin, 2002, 53-69.

[8] -, On the algebraic thickness and non-normality of Boolean functions, dans : Proc. "2003 IEEE Information Theory Workshop", Paris, 2003, 147-150.

[9] C. Carlet and P. Guillot, A characterization of binary bent functions, J. Combin. Theory Ser. A 76 (1996), 328-335.

[10] C. Carlet and A. Klapper, Upper bounds on the numbers of resilient functions and of bent functions, Lecture Notes dédiées à Philippe Delsarte, Springer, à paraître; une version abrégée est paru dans les Proceedings of the 23rd Symposium on Information Theory in the Benelux, Louvain-La-Neuve, 2002.

[11] F. Chabaud and S. Vaudenay, Links between differential and linear cryptanalysis, dans : Advances in Cryptology-EUROCRYPT 1994 (Perugia), Lecture Notes in Comput. Sci. 950, Springer, Berlin, 1995, 356-365.

[12] A. Dembo and O. Zeitouni, Large Deviations Techniques and Applications, Appl. Math. (New York) 38, Springer, New York, 1998.

[13] J. Dillon, Elementary Hadamard difference sets, thèse de doctorat, Univ. of Maryland, 1974.

[14] T. Erdélyi, Polynomials with Littlewood-type coefficient constraints, dans : Approximation Theory X, C. K. Chui, L. L. Schumaker and J. Stöckler (eds.), Vanderbilt Univ. Press, Nashville, TN, 2002, 153-196.

[15] P. Erdős, Some unsolved problems, Michigan Math. J. 4 (1957), 291-300. 
[16] C. Fontaine, Contribution à la recherche de fonctions booléennes hautement non linéaires et au marquage d'images en vue de la protection des droits d'auteur, thèse, Univ. Paris VI, 1998; http://www-rocq.inria.fr/codes/Caroline.Fontaine/THESE/ these.html.

[17] J.-P. Kahane, Some Random Series of Functions, 2nd ed., Cambridge Stud. Adv. Math. 5, Cambridge Univ. Press, Cambridge, 1985.

[18] B. Kashin and L. Tsafriri [L. Tzafriri], A lower bound for the maximum of a random process, Math. Notes 56 (1994), 1306-1308.

[19] P. Langevin, Les sommes de caractères et la formule de Poisson dans la théorie des codes, des séquences et des fonctions booléennes, Habilitation à Diriger les Recherches, Univ. de Toulon et du Var, 1999; http://www.univ-tln.fr/ langevin/.

[20] P. Langevin and P. Solé, Kernels and defaults, dans : Finite Fields: Theory, Applications and Algorithms, G. L. Mullen and R. C. Mullin (eds.), Contemp. Math. 225, Amer. Math. Soc., Providence, RI, 1999, 77-85.

[21] J. E. Littlewood, On polynomials $\sum^{n} \pm z^{m}, \sum^{n} e^{\alpha_{m} i} z^{m}, z=e^{\theta_{i}}$, J. London Math. Soc. 41 (1966), 367-376.

[22] W. Meier and O. Staffelbach, Nonlinearity criteria for cryptographic functions, dans: Advances in Cryptology-EUROCRYPT '89 (Houthalen), Lecture Notes in Comput. Sci. 434, Springer, Berlin, 1990, 549-562.

[23] D. Newman and J. Byrnes, The $L^{4}$ norm of a polynomial with coefficients \pm 1 , Amer. Math. Monthly 97 (1990), 42-45.

[24] K. Nyberg, Perfect nonlinear S-boxes, dans : Advances in Cryptology-EUROCRYPT '91 (Brighton), Lecture Notes in Comput. Sci. 547, Springer, Berlin, 1991, 378-386.

[25] D. Olejár and M. Stanek, On cryptographic properties of random Boolean functions, J. Universal Comput. Sci. 4 (1998), 705-717.

[26] N. Patterson and D. Wiedemann, The covering radius of the $\left(2^{15}, 16\right)$ Reed-Muller code is at least 16276 , IEEE Trans. Inform. Theory 29 (1983), 354-356.

[27] R. Salem and A. Zygmund, Some properties of trigonometric series whose terms have random signs, Acta Math. 91 (1954), 245-301.

[28] P. Stănică, Nonlinearity, local and global avalanche characteristics of balanced Boolean functions, Discrete Math. 248 (2002), 181-193.

[29] X. M. Zhang and Y. L. Zheng, GAC-the criterion for global avalanche characteristics of cryptographic functions, J. Universal Comput. Sci. 1 (1995), 320-337.

Institut de Mathématiques de Luminy

Case 907

163 Avenue de Luminy

13288 Marseille Cedex 9, France

E-mail: rodier@iml.univ-mrs.fr 